\title{
Ram Pressure Stripping of Spiral Galaxies in Clusters
}

\author{
Elke Schumacher, Gerhard Hensler \\ Institut für Theoretische Physik und Astrophysik, Universität Kiel, \\ Olshausenstr. 40, 24098 Kiel, Germany
}

\begin{abstract}
We investigate the process of ram pressure stripping by means of numerical simulations with a $2 \mathrm{D}$ hydrodynamical code. We present some first results of a set of simulations with varying galaxy velocities and ICM densities. We find that in typical cluster core environments disk galaxies lose a substantial amount of their gas, whereas in the outskirts of galaxy clusters the mass loss is quite small. Furthermore, the gas loss happens in two phases: In the initial phase gas is pushed out of regions where the ram pressure overcomes the gravitational restoring force; most of the overall gas loss happens in this phase. Afterwards the Kelvin-Helmholtz instability leads to a further mass loss at a small rate, that could be important on long timescales.
\end{abstract}

\section{Introduction}

The environment has an important influence on the evolution of galaxies. Comparative observations of spiral galaxies in clusters and of those in the field reveal mainly differences that are connected with their gas contents. E.g., cluster spirals often show HI deficiency, truncated $\mathrm{HI}$ and $\mathrm{H} \alpha$ disks, but undisturbed stellar disks. An overview for the Virgo cluster galaxies is given by Cayatte, van Gorkom, \& Kotanyi (1990). Another striking feature is revealed by comparing the galaxy populations in the field and in clusters. The field population is dominated by late type galaxies, whereas clusters contain a large fraction of early type galaxies. Especially S0 galaxies are mainly found in dense environments, indicating that there may be a process that turns gas rich spirals into gas poor S0 galaxies.

Besides tidal interactions and galaxy harassment the process of ram pressure stripping - the removal of a galaxy's gas due to its motion through the intracluster medium (ICM) - is a good candidate to explain these observations.

As one is mainly interested in the effect on the galaxy, the relative motion between the galaxy and the ICM is usually studied in the rest frame of the galaxy, where the ICM appears as a wind that flows past the galaxy. The relative motion applies the ram pressure $\sim \rho_{\mathrm{ICM}} v^{2}$ to the galactic interstellar medium (ISM), where $\rho_{\mathrm{ICM}}$ is the ICM density and $v$ is either the galaxy velocity (in the cluster rest frame) or the ICM "wind" velocity (in the galaxy rest frame). For a first estimate about the fate of the ISM one can compare the ram pressure with the restoring force of the galaxy's gravity (per unit area). For typical ICM and 
galaxy parameters this comparison favours the ram pressure and predicts that the galaxy should lose at least some of its ISM.

In the strict sense the term ram pressure stripping refers only to the mentioned interplay between the ram pressure and the galaxy's gravity. In addition, gas flows involve also turbulence, viscosity and instabilities like the KelvinHelmholtz $(\mathrm{KH})$ instability. These processes may play an important role in the flow of the ICM past the galaxy. Sometimes ISM loss due to these processes is referred to as turbulent and viscous stripping, but often (also here) these processes are included in the term ram pressure stripping, giving it a more general meaning.

In contrast to tidal interactions, only the gaseous disk but not the stellar disk should be disturbed by ram pressure stripping, which is the main criterion to distinguish these scenarios. The Virgo galaxy NGC4522 (see Kenney \& Koopmann 1999, 2001) is one of the best known individual examples of galaxies showing exactly these features of the stripping process.

\section{The model}

The simulation of the interaction between the galaxy and the ICM is done in the rest frame of the galaxy, that means that the ICM is flowing past the galaxy (like a wind).

We use a two-dimensional cylinder symmetrical hydrodynamical Eulerian grid code similar to that of Norman \& Winkler (1986). The gravitational potential resulting from all density distributions together is computed via an ADIsolver (Press et al. 1992).

Initially, the model galaxy consists of a Plummer-Kuzmin (PK) disk (containing a stellar and a gaseous component) and a dark matter (DM) halo of the form proposed by Burkert (1995). The rotation curve is flat. Parameters of the initial model are listed in table 1.

\begin{tabular}{ll} 
Table $1 . \quad$ Parameters of the model galaxy \\
\hline Disk \\
\hline total disk mass & $0.77 \cdot 10^{11} M_{\odot}$ \\
gas fraction & $10 \%$ \\
disk PK scale length $a$ & $4 \mathrm{kpc}$ \\
disk PK flatness $\frac{b}{a}$ & 0.05 \\
disk radius & $19 \mathrm{kpc}$ \\
maximal rotation velocity & $200 \mathrm{~km} \mathrm{~s}-1$ \\
\hline Dark Matter Halo & \\
\hline scale radius of dark matter profile $r_{\mathrm{DM}}$ & $30 \mathrm{kpc}$ \\
central dark matter density $\rho_{\mathrm{DM}}$ & $4.7 \cdot 10^{-3} M_{\odot} \mathrm{pc}^{-3}$ \\
total dark matter mass & $4 \cdot 10^{11} M_{\odot}$ \\
\hline \hline
\end{tabular}

Initially, the model galaxy is set up in equilibrium with the ICM at rest. When the simulation is started, the ICM inflow velocity is increased over the first 50Myr to its final value, then it is kept constant. 


\section{Results}

In the first set of simulations that is presented here the ICM temperature was set to $10^{7} \mathrm{~K}$ in all runs, which corresponds to an ICM sound speed of $c_{\mathrm{ICM}}=477 \mathrm{~km} \mathrm{~s}^{-1}$. The simulations are done at solar metalicity. We explored the parameter space for the ICM wind in the subsonic range, varying the ICM particle density $\left(n_{\mathrm{ICM}}\right)$ and the ICM velocity $\left(v_{\mathrm{ICM}}\right)$. Table 2 gives an overview over the simulation runs.

Table 2. ICM wind parameters for the simulations

\begin{tabular}{ccccc}
\hline & \multicolumn{4}{c}{$n_{\mathrm{ICM}} / \mathrm{cm}^{-3}$} \\
& \multicolumn{1}{c}{$10^{-3}$} & $10^{-3.5}$ & $10^{-4}$ \\
\hline \multirow{2}{*}{$v_{\mathrm{ICM}} / c_{\mathrm{ICM}}$} & 0.3 & $\times$ & $\times$ & - \\
& 0.5 & $\times$ & - & $\times$ \\
& 0.7 & $\times$ & $\times$ & $\times$ \\
\hline \hline
\end{tabular}

The behaviour of the galaxy in the ICM wind is in principle the same in all simulations. As soon as the galaxy is exposed to the wind, all gas of the outer disk that is not bound tightly enough by the gravitational potential is practically "pushed out" of the disk during the first few hundred Myrs. Figure 1 shows the density distribution of ICM and ISM in the initial state and at the end of this first phase for the simulation with the strongest ICM wind. This first

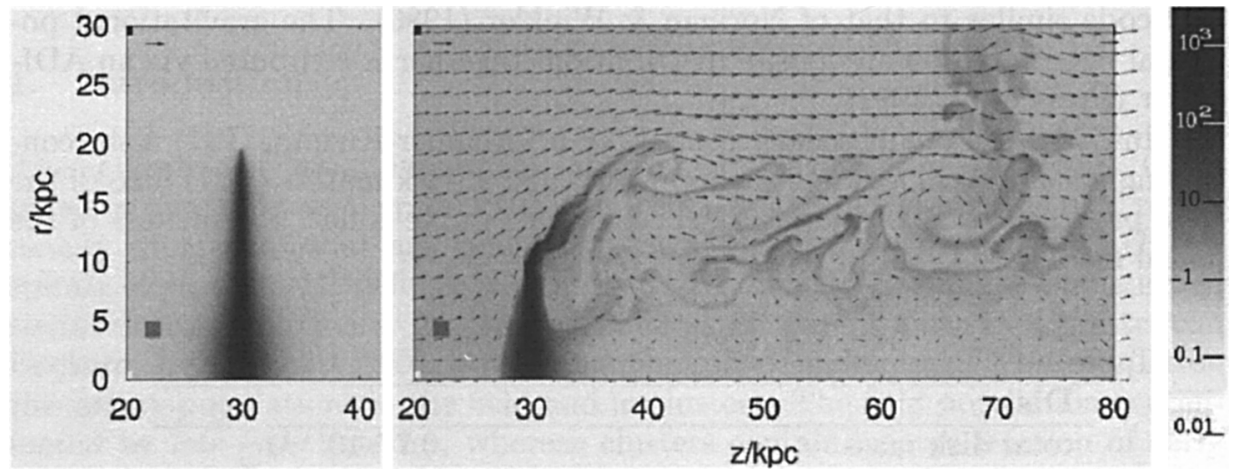

Figure 1. Snapshot of the simulation with $n_{\mathrm{ICM}}=10^{-3} \mathrm{~cm}^{-3}$, $v_{\mathrm{ICM}} / c_{\mathrm{ICM}}=0.7$, at beginning and at $700 \mathrm{Myr}$. The gas density is shown on a logarithmic gray-scale, the scale on the right is in units of $10^{-3} \mathrm{~cm}^{-3}$. The arrows show the velocity field, the bold arrows in the upper corners indicate the original $v_{\text {ICM }}$. The dark gray rectangle on the upstream side of the galaxy demonstrates the spatial resolution, it represents the size of $10 \times 10$ grid cells.

phase is followed by a long period where gas is still lost from the disk by the $\mathrm{KH}$ instability. In this phase the mass loss rate is small $\left(<0.5 M_{\odot} \mathrm{yr}^{-1}\right)$, but this period continues for the rest of the simulation, which is up to a few Gyrs.

The time evolution of the mass of the gas remaining in the galactic disk is shown in figure 2 for three different simulations. The first phase with the rapid 


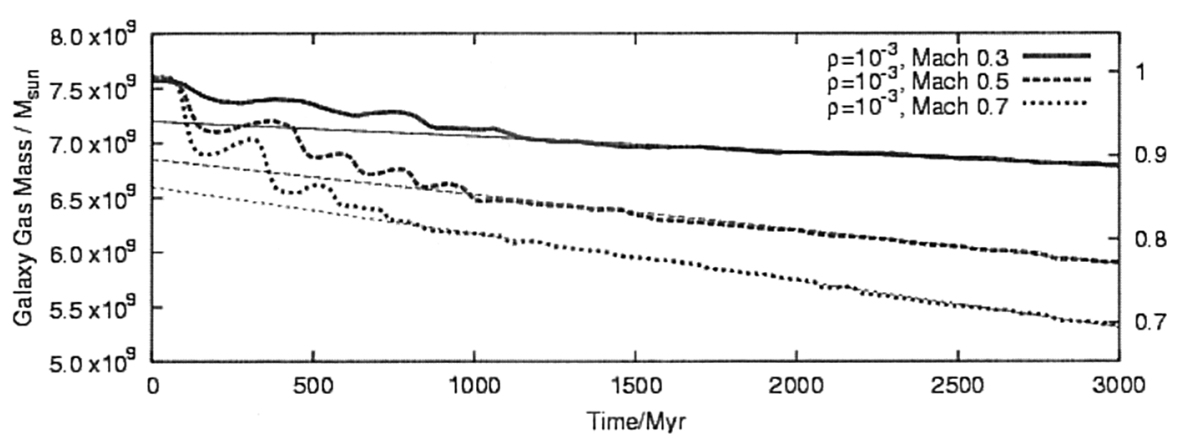

Figure 2. Time evolution of the mass of the remaining gas disk for three different ICM wind cases ( $\rho$ in units of $\mathrm{cm}^{-3}$ and the Mach number $v_{\mathrm{ICM}} / c_{\mathrm{ICM}}$ are given in the plot).

mass loss and the long $\mathrm{KH}$ phase can be clearly distinguished. Interestingly the mass loss rate in the Kelvin-Helmholtz phase is constant. The transition between the two phases happens later in the simulations with a slow ICM wind.

One question one wants to answer with such simulations is: How much ISM will the galaxy lose if it moves with velocity $v$ through the ICM of density $\rho_{\mathrm{ICM}}$ ? From our simulations this prediction is not straightforward to make, as neither the radius of the remaining gas disk nor its mass do converge during the simulation time. That's why we decided to measure these quantities in two different versions: Firstly, we want to separate the influence of the initial "pushing" phase. Hence we measure the mass and radius of the remaining gas disk after $1.25 \mathrm{Gyr}$ in all runs. At that time the initial phase is definitely finished, and the effect of the KH phase is still small due to its low gas loss rate. Secondly, we want to compare the fate of the galaxy after it has passed a cluster core, that is after it has flown a distance of about $1 \mathrm{Mpc}$. Of course slower galaxies need longer to move this distance.

As so far only a small number of simulation runs has been performed and hence the parameter space is not yet sampled sufficiently, we skip a detailed comparison between analytical estimates of the stripping radius and the remaining gas mass with the numerical result. The analytical estimate does roughly agree with the numerical result, but to understand the differences better more simulation runs are necessary.

Something that can already be done even with this small set of simulations is to search for a correlation between the ICM wind strength and the remaining gas disk. The wind strength of course depends on $\rho_{\mathrm{ICM}}$ and $v_{\mathrm{ICM}}$ combined. One meaningful combination could be the ram pressure $\rho_{\mathrm{ICM}} v_{\mathrm{ICM}}^{2}$, another might be the momentum density $\rho_{\mathrm{ICM}} v_{\mathrm{ICM}}$ carried by the ICM wind. In figure 3 we plot for each simulation the stripping radius and the mass of the remaining ISM as a function of the ram pressure $\rho_{\mathrm{ICM}} v_{\mathrm{ICM}}^{2}$, both for the "measurement" after $1.25 \mathrm{Gyr}$ and after $1 \mathrm{Mpc}$. For the range of more than one order of magnitude in ram pressure covered by the simulations so far, both the mass and radius of the remaining gas disk are tightly related to the ram pressure via power laws. We also checked if the mass and radius of the remaining gas disk are correlated 

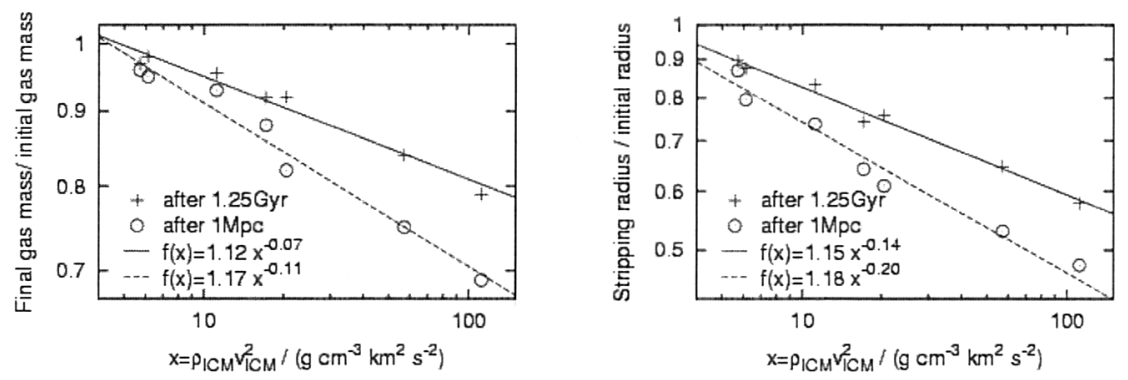

Figure 3. Mass and radius of the remaining gas disk (in units of initial values) as a function of the ram pressure (see also text).

with the momentum density $\rho_{\mathrm{ICM}} v_{\mathrm{ICM}}$ carried by the wind. We find a similar power law correlation, but it is not as tight as the one to the ram pressure. Of course also these results have to be confirmed with a larger set of simulations.

\section{Conclusions and future work}

We have performed a first set of simulations of ram pressure stripping of disk galaxies in galaxy clusters. The simulations so far model a rather slow passage of a normal spiral galaxy through the central region of a rich cluster of galaxies (like the Virgo cluster). The simulations predict that the galaxy will lose a substantial amount of its gas unless it moves through a low density ICM or it is very slow. The gas loss happens in two phases. During the first few hundred Myrs ram pressure stripping in the strict sense causes the main part of the overall gas loss. In the following few Gyrs turbulent and viscous stripping (the $\mathrm{KH}$ phase) continues with a small mass loss rate of less than $0.5 M_{\odot} \mathrm{yr}^{-1}$.

The next step to continue this work is to extend the parameter study, especially to include higher, more realistic galaxy velocities, both in the subsonic and in the supersonic range.

In order to observe the stripping process directly, the stripped material must form clumps of sufficient density and size. In the simulations so far these conditions are only met during the initial phase where the gas loss rate is highest. However, due to the brevity of this phase the probability to detect a galaxy in this phase is small. This is in agreement with the small number of direct observations. On the other hand, up to now the influence of heating, cooling and thermal conduction is neglected. These processes may play an important role especially for the fate of the stripped material, hence they should be included in future simulations.

The interaction between the ICM and the ISM of galaxies moving through clusters plays definitely an important role for the evolution of galaxies. A parametrisation of the impact of this process like from figure 3 can be very useful for cosmological simulations that study the evolution of whole populations of galaxies and track the evolution of their gas contents, star formation and Hubble types. 
Acknowledgments. This work is supported by the DFG under grant HE$1487 / 30$.

\section{References}

Burkert, A. 1995, ApJ, 447, 25L

Cayatte, V., van Gorkom, J. H., \& Kotanyi, C. 1990, AJ, 100, 604

Kenney, J. D. P. \& Koopmann, R. A. 1999, AJ, 117, 181

Kenney, J. D. P. \& Koopmann, R. A. 2001, In ASP Conf. Ser. Vol. 240, Gas and Galaxy Evolution, ed. J. E. Hibbard, M. P. Rupen \& J. J. van Gorkom (San Francisco:ASP), 577

Norman, M. L. \& Winkler, K.-H. A. 1986, In NATO Advanced Research Workshop, Astrophysical Radiation Hydrodynamics, ed. K.-H. A. Winkler \& M. L. Norman (Dortrecht: Reidel), 187

Press, W.H., Teukolsky S.A., Vetterling W.T., \& Flannery B.P. 1992, Numerical Recipes, Cambridge University Press 